\title{
$\triangle$-Convergence Problems for Asymptotically Nonexpansive Mappings in CAT(0) Spaces
}

\author{
Luo Yi Shi, ${ }^{1}$ Ru Dong Chen, ${ }^{1}$ and Yu Jing $W^{2}{ }^{2}$ \\ ${ }^{1}$ Department of Mathematics, Tianjin Polytechnic University, Tianjin 300387, China \\ ${ }^{2}$ Tianjin Vocational Institute, Tianjin 300410, China \\ Correspondence should be addressed to Ru Dong Chen; chenrd@tjpu.edu.cn
}

Received 23 January 2013; Accepted 17 February 2013

Academic Editor: Yisheng Song

Copyright (c) 2013 Luo Yi Shi et al. This is an open access article distributed under the Creative Commons Attribution License, which permits unrestricted use, distribution, and reproduction in any medium, provided the original work is properly cited.

\begin{abstract}
New $\triangle$-convergence theorems of iterative sequences for asymptotically nonexpansive mappings in CAT(0) spaces are obtained. Consider an asymptotically nonexpansive self-mapping $T$ of a closed convex subset $C$ of a CAT( 0$)$ space $X$. Consider the iteration process $\left\{x_{n}\right\}$, where $x_{0} \in C$ is arbitrary and $x_{n+1}=\alpha_{n} x_{n} \oplus\left(1-\alpha_{n}\right) T^{n} y_{n}$ or $x_{n+1}=\alpha_{n} T^{n} x_{n} \oplus\left(1-\alpha_{n}\right) y_{n}, y_{n}=\beta_{n} x_{n} \oplus\left(1-\beta_{n}\right) T^{n} x_{n}$ for $n \geq 1$, where $\left\{\alpha_{n}\right\},\left\{\beta_{n}\right\} \subset(0,1)$. It is shown that under certain appropriate conditions on $\alpha_{n}, \beta_{n},\left\{x_{n}\right\} \triangle$-converges to a fixed point of $T$.
\end{abstract}

\section{Introduction and Preliminaries}

Let $C$ be a nonempty subset of a metric space $(X, d)$. A mapping $T: C \rightarrow C$ is a contraction if there exists $k \in[0,1)$ such that for all $x, y \in C$, we have $d(T x, T y)<$ $k d(x, y)$. It is said to be nonexpansive if for all $x, y \in C$, we have $d(T x, T y) \leq d(x, y)$. T is said to be asymptotically nonexpansive if there exists a sequence $\left\{k_{n}\right\} \in[1, \infty)$ with $k_{n} \rightarrow 1$ such that $d\left(T^{n} x, T^{n} y\right) \leq k_{n} d(x, y)$ for all integers $n \geq 1$ and all $x, y \in C$. Clearly, every contraction mapping is nonexpansive and every nonexpansive mapping is asymptotically nonexpansive with sequence $k_{n}=1$, for all $n \geq 1$. There are, however, asymptotically nonexpansive mappings which are not nonexpansive (see, e.g., [1]). As a generalization of the class of nonexpansive mappings, the class of asymptotically nonexpansive mappings was introduced by Goebel and Kirk [2] in 1972 and has been studied by several authors (see, e.g., [3-5]). Goebel and Kirk proved that if $C$ is a nonempty closed convex and bounded subset of a uniformly convex Banach space (more general than a Hilbert space, i.e., CAT(0) space), then every asymptotically nonexpansive self-mapping of $C$ has a fixed point. The weak and strong convergence problems to fixed points of nonexpansive and asymptotically nonexpansive mappings have been studied by many authors.

We will denote by $F(T)$ the set of fixed points of $T$. In 1967, Halpern [6] introduced an explicit iterative scheme for a nonexpansive mapping $T$ on a subset $C$ of a Hilbert space by taking any point $u, x_{1} \in C$ and defined the iterative sequence $\left\{x_{n}\right\}$ by

$$
x_{n+1}=\alpha_{n} u+\left(1-\alpha_{n}\right) T x_{n}, \quad \text { for } n \geq 1,
$$

where $\alpha_{n} \in[0,1]$. He pointed out that under certain appropriate conditions on $\alpha_{n},\left\{x_{n}\right\}$ converges strongly to a fixed point of T. In 1994, Tan and Xu [7] introduced the following iterative scheme for asymptotically nonexpansive mapping on uniformly convex Banach space:

$$
\begin{gathered}
x_{0} \in C, \\
x_{n+1}=\alpha_{n} f\left(x_{n}\right)+\left(1-\alpha_{n}\right) T^{n} y_{n}, \quad n \geq 0, \\
y_{n}=\gamma_{n} x_{n}+\left(1-\gamma_{n}\right) T^{n} x_{n}, \quad n \geq 0,
\end{gathered}
$$

where $\left\{\alpha_{n}\right\},\left\{\gamma_{n}\right\} \subseteq(0,1)$. They proved that under certain appropriate conditions on $\alpha_{n}, \gamma_{n},\left\{x_{n}\right\}$ converges weakly to a fixed point of $T$.

In 2012, we [8] studied the viscosity approximation methods for nonexpansive mappings on CAT(0) space. For a contraction $f$ on $C$, consider the iteration process $\left\{x_{n}\right\}$, where $x_{0} \in C$ is arbitrary and

$$
x_{n+1}=\alpha_{n} f\left(x_{n}\right) \oplus\left(1-\alpha_{n}\right) T x_{n},
$$


for $n \geq 1$, where $\left\{\alpha_{n}\right\} \subset(0,1)$. We proved that under certain appropriate conditions on $\alpha_{n},\left\{x_{n}\right\}$ converges strongly to a fixed point of $T$ which solves some variational inequality.

The purpose of this paper is to study the iterative scheme defined as follows: consider an asymptotically nonexpansive self-mapping $T$ of a closed convex subset $C$ of a CAT(0) space $X$ with coefficient $k_{n}$. consider the iteration process $\left\{x_{n}\right\}$, where $x_{0} \in C$ is arbitrary and

$$
\begin{gathered}
x_{n+1}=\alpha_{n} x_{n} \oplus\left(1-\alpha_{n}\right) T^{n} y_{n}, \\
y_{n}=\beta_{n} x_{n} \oplus\left(1-\beta_{n}\right) T^{n} x_{n},
\end{gathered}
$$

or

$$
\begin{gathered}
x_{n+1}=\alpha_{n} T^{n} x_{n} \oplus\left(1-\alpha_{n}\right) y_{n}, \\
y_{n}=\beta_{n} x_{n} \oplus\left(1-\beta_{n}\right) T^{n} x_{n},
\end{gathered}
$$

for $n \geq 1$, where $\left\{\alpha_{n}\right\},\left\{\beta_{n}\right\} \subset(0,1)$. We show that $\left\{x_{n}\right\} \triangle$ converges to a fixed point of $T$ under certain appropriate conditions on $\alpha_{n}, \beta_{n}$, and $k_{n}$.

We now collect some elementary facts about CAT(0) spaces which will be used in the proofs of our main results.

Lemma 1. Let $X$ be a CAT(0) space. Then, one has the following:

(i) (see [9, Lemma 2.4]) for each $x, y, z \in X$ and $t \in[0,1]$, one has

$$
d((1-t) x \oplus t y, z) \leq(1-t) d(x, z)+t d(y, z),
$$

(ii) (see [10]) for each $x, y, z \in X$ and $t, s \in[0,1]$ one has

$$
d((1-t) x \oplus t y,(1-s) x \oplus s y) \leq|t-s| d(x, y),
$$

(iii) (see [5, Lemma 3]) for each $x, y, z \in X$ and $t \in[0,1]$, one has

$$
d((1-t) z \oplus t x,(1-t) z \oplus t y) \leq t d(x, y),
$$

(iv) (see [9]) for each $x, y, z \in X$ and $t \in[0,1]$, one has

$$
\begin{aligned}
& d^{2}((1-t) x \oplus t y, z) \\
& \quad \leq t d^{2}(x, z)+(1-t) d^{2}(y, z)-t(1-t) d^{2}(x, y) .
\end{aligned}
$$

Let $X$ be a complete CAT(0) space and let $\left\{x_{n}\right\}$ be a bounded sequence in a complete $X$ and for $x \in X$ set

$$
r\left(x,\left\{x_{n}\right\}\right)=\limsup _{n \rightarrow \infty} d\left(x, x_{n}\right) .
$$

The asymptotic radius $r\left(\left\{x_{n}\right\}\right)$ of $\left\{x_{n}\right\}$ is given by

$$
r\left(\left\{x_{n}\right\}\right)=\inf \left\{r\left(x,\left\{x_{n}\right\}\right): x \in X\right\},
$$

and the asymptotic center $A\left(\left\{x_{n}\right\}\right)$ of $\left\{x_{n}\right\}$ is the set

$$
A\left(\left\{x_{n}\right\}\right)=\left\{x \in X: r\left(x,\left\{x_{n}\right\}\right)=r\left(\left\{x_{n}\right\}\right)\right\} .
$$

It is known (see, e.g., [11, Proposition 7]) that in a CAT(0) space, $A\left(\left\{x_{n}\right\}\right)$ consists of exactly one point.

A sequence $\left\{x_{n}\right\}$ in $X$ is said to $\triangle$-converge to $x \in X$ if $x$ is the unique asymptotic center of $\left\{u_{n}\right\}$ for every subsequence $\left\{u_{n}\right\}$ of $\left\{x_{n}\right\}$. In this case, we write $\triangle-\lim _{n} x_{n}=x$ and call $x$ the $\triangle$-limit of $\left\{x_{n}\right\}$.
Lemma 2. Assume that $X$ is a CAT(0) space. Then, one has the following:

(i) (see [12]) every bounded sequence in $X$ has a $\triangle$ convergent subsequence;

(ii) (see [13]) if $K$ is a closed convex subset of $X$ and $T: K \rightarrow X$ is an asymptotically nonexpansive mapping, then the conditions $\left\{x_{n}\right\} \triangle$-converge to $x$ and $d\left(x_{n}, T\left(x_{n}\right)\right) \rightarrow 0$, imply $x \in K$ and $x \in F(T)$.

Lemma 3 (see $[14,15]$ ). Let $\left\{a_{n}\right\},\left\{b_{n}\right\}$, and $\left\{c_{n}\right\}$ be three nonnegative real sequences satisfying the following condition:

$$
a_{n+1} \leq\left(1+b_{n}\right) a_{n}+c_{n}, \quad \forall n \geq n_{0}
$$

where $n_{0}$ is some nonnegative integer, $\sum_{n=1}^{\infty} b_{n}<\infty, \sum_{n=1}^{\infty} c_{n}<$ $\infty$. Then the limit $\lim _{n \rightarrow \infty} a_{n}$ exists.

\section{2. $\triangle$-Convergence of the Iteration Sequences}

In this section, we will study the $\triangle$-convergence of the iteration sequence for asymptotically nonexpansive mappings in $\mathrm{CAT}(0)$ spaces.

Suppose that $X$ be a CAT(0) space, $C$ a closed convex subset of $X$, and $T: C \rightarrow C$ an asymptotically nonexpansive mapping with coefficient $k_{n}$. Firstly, we consider the iteration process:

$$
\begin{gathered}
x_{0} \in C, \\
x_{n+1}=\alpha_{n} x_{n} \oplus\left(1-\alpha_{n}\right) T^{n} y_{n}, \quad n \geq 0, \\
y_{n}=\beta_{n} x_{n} \oplus\left(1-\beta_{n}\right) T^{n} x_{n}, \quad n \geq 0,
\end{gathered}
$$

where $\left\{\alpha_{n}\right\},\left\{\beta_{n}\right\} \subseteq(0,1)$ and $k_{n}$ satisfy the following.

(i) There exist positive integers $n_{0}, n_{1}$, and $\delta>0,0<b<$ $\min \{1,1 / L\}$, where $L=\sup _{n} k_{n}$, such that

$$
\begin{gathered}
0<\delta<\alpha_{n}<1-\delta, \quad n \geq n_{0}, \\
0<1-\beta_{n}<b, \quad n \geq n_{1},
\end{gathered}
$$

(ii) Consider $\sum_{n=1}^{\infty}\left(k_{n}^{2}-1\right)<\infty$.

We will prove that $\left\{x_{n}\right\} \triangle$-converges to a fixed point of $T$.

Lemma 4. Let $X$ be a CAT(0) space, $C$ a closed convex subset of $X, T: C \rightarrow C$ an asymptotically nonexpansive mapping with coefficient $k_{n}$, and $\sum_{n=1}^{\infty}\left(k_{n}^{2}-1\right)<\infty$. If $F(T) \neq \emptyset,\left\{\alpha_{n}\right\},\left\{\beta_{n}\right\} \subseteq$ $(0,1)$. Let $x_{0} \in C,\left\{x_{n}\right\}$ be generated by $x_{n+1}=\alpha_{n} x_{n} \oplus(1-$ $\left.\alpha_{n}\right) T^{n} y_{n}, y_{n}=\beta_{n} x_{n} \oplus\left(1-\beta_{n}\right) T^{n} x_{n}, n \geq 0$. Then the limit $\lim _{n \rightarrow \infty} d\left(x_{n}, p\right)$ exists for all $p \in F(T)$. 
Proof. Taking $p \in F(T)$, we have

$$
\begin{aligned}
d\left(x_{n+1}, p\right)= & d\left(\alpha_{n} x_{n} \oplus\left(1-\alpha_{n}\right) T^{n} y_{n}, p\right) \\
\leq & \alpha_{n} d\left(x_{n}, p\right)+\left(1-\alpha_{n}\right) d\left(T^{n} y_{n}, p\right) \\
\leq & \alpha_{n} d\left(x_{n}, p\right)+\left(1-\alpha_{n}\right) k_{n} d\left(y_{n}, p\right) \\
\leq & \alpha_{n} d\left(x_{n}, p\right) \\
& +\left(1-\alpha_{n}\right) k_{n}\left\{\beta_{n} d\left(x_{n}, p\right)\right. \\
\leq & \left.\alpha_{n} d\left(x_{n}, p\right)+\left(1-\beta_{n}\right) d\left(T^{n} x_{n}, p\right)\right\} \\
& +\left(1-\alpha_{n}\right) k_{n}\left\{\beta_{n} d\left(x_{n}, p\right)\right. \\
= & \left\{1+\left(1-\alpha_{n}\right)\left(k_{n}-1\right)\right. \\
& \left.\times\left[k_{n}\left(1-\beta_{n}\right)+1\right]\right\} d\left(x_{n}, p\right) \\
\leq & \left\{1+\left(k_{n}^{2}-1\right)\right\} d\left(x_{n}, p\right) .
\end{aligned}
$$

By Lemma 3, we can get that $\lim _{n \rightarrow \infty} d\left(x_{n}, p\right)$ exists.

Remark 5. The above lemma implies that $\left\{x_{n}\right\}$ is bounded and so is the sequence $\left\{T x_{n}\right\}$. Moreover, let $L=\sup _{n} k_{n}$, then we have

$$
\begin{gathered}
d\left(T^{n} x_{n}, p\right) \leq k_{n} d\left(x_{n}, p\right) \leq \operatorname{Ld}\left(x_{n}, p\right), \\
d\left(y_{n}, p\right) \leq \beta_{n} d\left(x_{n}, p\right)+\left(1-\beta_{n}\right) d\left(T^{n} x_{n}, p\right) \\
\leq \operatorname{Ld}\left(x_{n}, p\right) \\
d\left(T^{n} y_{n}, p\right) \leq k_{n} d\left(y_{n}, p\right) \leq L^{2} d\left(x_{n}, p\right) .
\end{gathered}
$$

It follows that the sequences $\left\{T^{n} x_{n}\right\},\left\{y_{n}\right\},\left\{T^{n} y_{n}\right\}$ are bounded.

Proposition 6. Let $X$ be a CAT(0) space, $C$ a closed convex subset of $X$, and $T: C \rightarrow C$ an asymptotically nonexpansive mapping with coefficient $k_{n}$. If $F(T) \neq \emptyset,\left\{\alpha_{n}\right\},\left\{\beta_{n}\right\} \subseteq(0,1)$. Let $x_{0} \in C,\left\{x_{n}\right\}$ be generated by $x_{n+1}=\alpha_{n} x_{n} \oplus\left(1-\alpha_{n}\right) T^{n} y_{n}$, $y_{n}=\beta_{n} x_{n} \oplus\left(1-\beta_{n}\right) T^{n} x_{n}, n \geq 0$. Then under the hypotheses (i) and (ii), one can get that $\lim _{n \rightarrow \infty} d\left(x_{n}, T^{n} y_{n}\right)=0$.

Proof. By the assumption, $F(T)$ is nonempty. Take $p \in F(T)$, by Lemma 1(iv), we have

$$
\begin{aligned}
d^{2}\left(x_{n+1}, p\right)= & d^{2}\left(\alpha_{n} x_{n} \oplus\left(1-\alpha_{n}\right) T^{n} y_{n}, p\right) \\
\leq & \alpha_{n} d^{2}\left(x_{n}, p\right)+\left(1-\alpha_{n}\right) d^{2}\left(T^{n} y_{n}, p\right) \\
& \quad-\alpha_{n}\left(1-\alpha_{n}\right) d^{2}\left(x_{n}, T^{n} y_{n}\right) \\
\leq & d^{2}\left(x_{n}, p\right)+\left(1-\alpha_{n}\right)\left\{d^{2}\left(T^{n} y_{n}, p\right)\right. \\
& \left.-d^{2}\left(y_{n}, p\right)\right\}
\end{aligned}
$$

$$
\begin{aligned}
&+\left(1-\alpha_{n}\right)\left\{d^{2}\left(y_{n}, p\right)-d^{2}\left(x_{n}, p\right)\right\} \\
&- \alpha_{n}\left(1-\alpha_{n}\right) d^{2}\left(x_{n}, T^{n} y_{n}\right), \\
& d^{2}\left(y_{n}, p\right)-d^{2}\left(x_{n}, p\right) \\
&=d^{2}\left(\beta_{n} x_{n} \oplus\left(1-\beta_{n}\right) T^{n} x_{n}, p\right)-d^{2}\left(x_{n}, p\right) \\
& \leq \beta_{n} d^{2}\left(x_{n}, p\right)+\left(1-\beta_{n}\right) d^{2}\left(T^{n} x_{n}, p\right) \\
& \quad-\beta_{n}\left(1-\beta_{n}\right) d^{2}\left(x_{n}, T^{n} x_{n}\right)-d^{2}\left(x_{n}, p\right) \\
& \leq \beta_{n} d^{2}\left(x_{n}, p\right)+\left(1-\beta_{n}\right) d^{2}\left(T^{n} x_{n}, p\right) \\
& \quad-d^{2}\left(x_{n}, p\right),
\end{aligned}
$$

which implies that

$$
\begin{aligned}
d^{2}\left(y_{n}, p\right)-d^{2}\left(x_{n}, p\right) & \leq\left(1-\beta_{n}\right)\left[d^{2}\left(T^{n} x_{n}, p\right)-d^{2}\left(x_{n}, p\right)\right] \\
& \leq\left(1-\beta_{n}\right)\left(k_{n}^{2}-1\right) d^{2}\left(x_{n}, p\right) .
\end{aligned}
$$

Therefore, we have

$$
\begin{aligned}
d^{2}\left(x_{n+1}, p\right) \leq & d^{2}\left(x_{n}, p\right)+\left(1-\alpha_{n}\right)\left(k_{n}^{2}-1\right) d^{2}\left(y_{n}, p\right) \\
& +\left(1-\alpha_{n}\right)\left(1-\beta_{n}\right)\left(k_{n}^{2}-1\right) d^{2}\left(x_{n}, p\right) \\
& -\alpha_{n}\left(1-\alpha_{n}\right) d^{2}\left(x_{n}, T^{n} y_{n}\right) .
\end{aligned}
$$

Since $\left\{x_{n}\right\}$ and $\left\{y_{n}\right\}$ are bounded and $0<\delta<\alpha_{n}<1-\delta$ for all $n \geq n_{0}$. we have

$$
\begin{aligned}
\delta^{2} d^{2}\left(x_{n}, T^{n} y_{n}\right) \leq & d^{2}\left(x_{n}, p\right)-d^{2}\left(x_{n+1}, p\right) \\
& +\left(1-\alpha_{n}\right)\left(k_{n}^{2}-1\right) d^{2}\left(y_{n}, p\right) \\
& +\left(1-\alpha_{n}\right)\left(1-\beta_{n}\right)\left(k_{n}^{2}-1\right) d^{2}\left(x_{n}, p\right) .
\end{aligned}
$$

By the conditions (i) and (ii), we have

$$
\sum_{n=1}^{\infty} \delta^{2} d^{2}\left(x_{n}, T^{n} y_{n}\right)<\infty
$$

which implies that

$$
\lim _{n \rightarrow \infty} d^{2}\left(x_{n}, T^{n} y_{n}\right)=0
$$

Theorem 7. Let $X$ be a CAT(0) space, $C$ a closed convex subset of $X$, and $T: C \rightarrow C$ an asymptotically nonexpansive mapping with coefficient $k_{n}$. If $F(T) \neq \emptyset,\left\{\alpha_{n}\right\},\left\{\beta_{n}\right\} \subseteq(0,1)$. Let $x_{0} \in C,\left\{x_{n}\right\}$ be generated by $x_{n+1}=\alpha_{n} x_{n} \oplus\left(1-\alpha_{n}\right) T^{n} y_{n}, y_{n}=$ $\beta_{n} x_{n} \oplus\left(1-\beta_{n}\right) T^{n} x_{n}, n \geq 0$. Then under the hypotheses ( $\left.i\right)$ and (ii), one can get that $\left\{x_{n}\right\} \triangle$-converges to a fix point of T. 
Proof. We first show that $\lim _{n \rightarrow \infty} d\left(x_{n}, T^{n} x_{n}\right)=0$. Indeed

$$
\begin{aligned}
d\left(x_{n}, y_{n}\right) & =d\left(x_{n}, \beta_{n} x_{n} \oplus\left(1-\beta_{n}\right) T^{n} x_{n}\right) \\
& \leq\left(1-\beta_{n}\right) d\left(x_{n}, T^{n} x_{n}\right) \\
& \leq\left(1-\beta_{n}\right)\left\{d\left(x_{n}, T^{n} y_{n}\right)+d\left(T^{n} y_{n}, T^{n} x_{n}\right)\right\} \\
& \leq\left(1-\beta_{n}\right)\left\{d\left(x_{n}, T^{n} y_{n}\right)+\operatorname{Ld}\left(y_{n}, x_{n}\right)\right\}
\end{aligned}
$$

it follows that

$$
\left[1-L\left(1-\beta_{n}\right)\right] d\left(x_{n}, y_{n}\right) \leq\left(1-\beta_{n}\right) d\left(x_{n}, T^{n} y_{n}\right) .
$$

By the conditions (i) and (ii) and Proposition 6, we get $\lim _{n \rightarrow \infty} d\left(x_{n}, y_{n}\right)=0$.

And then,

$$
\begin{aligned}
d\left(x_{n}, T^{n} x_{n}\right) & \leq d\left(x_{n}, T^{n} y_{n}\right)+d\left(T^{n} y_{n}, T^{n} x_{n}\right) \\
& \leq d\left(x_{n}, T^{n} y_{n}\right)+\operatorname{Ld}\left(y_{n}, x_{n}\right) .
\end{aligned}
$$

By Proposition 6, we get that $\lim _{n \rightarrow \infty} d\left(x_{n}, T^{n} x_{n}\right)=0$.

We claim that $\lim _{n \rightarrow \infty} d\left(x_{n}, T x_{n}\right)=0$. Indeed we have

$$
\begin{aligned}
& d\left(y_{n}, T^{n} x_{n}\right)=d\left(\beta_{n} x_{n} \oplus\left(1-\beta_{n}\right) T^{n} x_{n}, T^{n} x_{n}\right) \\
& \leq \beta_{n} d\left(x_{n}, T^{n} x_{n}\right) \longrightarrow 0 . \\
& d\left(x_{n+1}, x_{n}\right)=d\left(\alpha_{n} x_{n} \oplus\left(1-\alpha_{n}\right) T^{n} y_{n}, x_{n}\right) \\
& \leq\left(1-\alpha_{n}\right) d\left(x_{n}, T^{n} y_{n}\right) \longrightarrow 0 . \\
& d\left(x_{n-1}, T^{n-1} x_{n}\right) \leq d\left(x_{n-1}, T^{n-1} x_{n-1}\right) \\
& +d\left(T^{n-1} x_{n-1}, T^{n-1} x_{n}\right) \\
& \leq d\left(x_{n-1}, T^{n-1} x_{n-1}\right)+\operatorname{Ld}\left(x_{n-1}, x_{n}\right) \longrightarrow 0 . \\
& d\left(x_{n}, T^{n-1} x_{n}\right) \leq d\left(\alpha_{n-1} x_{n-1}\right. \\
& \left.\oplus\left(1-\alpha_{n-1}\right) T^{n-1} y_{n-1}, T^{n-1} x_{n}\right) \\
& \leq \alpha_{n-1} d\left(x_{n-1}, T^{n-1} x_{n}\right) \\
& +\left(1-\alpha_{n-1}\right) d\left(T^{n-1} y_{n-1}, T^{n-1} x_{n}\right) \\
& \leq \alpha_{n-1} d\left(x_{n-1}, T^{n-1} x_{n}\right) \\
& +\left(1-\alpha_{n-1}\right) \operatorname{Ld}\left(y_{n-1}, x_{n}\right) \\
& \leq \alpha_{n-1} d\left(x_{n-1}, T^{n-1} x_{n}\right) \\
& +\left(1-\alpha_{n-1}\right) L\left[d\left(y_{n-1}, x_{n-1}\right)\right. \\
& \left.+d\left(x_{n-1}, x_{n}\right)\right] \longrightarrow 0 \text {. }
\end{aligned}
$$

Thus,

$$
\begin{aligned}
d\left(x_{n}, T x_{n}\right) & \leq d\left(x_{n}, T^{n} x_{n}\right)+d\left(T^{n} x_{n}, T x_{n}\right) \\
& \leq d\left(x_{n}, T^{n} x_{n}\right)+L d\left(T^{n-1} x_{n}, x_{n}\right) \longrightarrow 0
\end{aligned}
$$

Since $\left\{x_{n}\right\}$ is bounded, we may assume that $\left\{x_{n}\right\} \triangle$ converges to a point $\widehat{x}$. By Lemma 2 , we have $\widehat{x} \in F(T)$.

Next we will consider another iteration process:

$$
\begin{gathered}
x_{0} \in C, \\
x_{n+1}=\alpha_{n} T^{n} x_{n} \oplus\left(1-\alpha_{n}\right) y_{n}, \quad n \geq 0, \\
y_{n}=\beta_{n} x_{n} \oplus\left(1-\beta_{n}\right) T^{n} x_{n}, \quad n \geq 0,
\end{gathered}
$$

where $\left\{\alpha_{n}\right\},\left\{\beta_{n}\right\} \subseteq(0,1)$, and $k_{n}$ satisfy the following

(H1) There exist positive integers $n_{0}$ and $\delta>0$, such that

$$
\begin{gathered}
0<\delta<\alpha_{n}<1-\delta, \quad n \geq n_{0} ; \\
1-\beta_{n} \longrightarrow 0
\end{gathered}
$$

(H2) $\sum_{n=1}^{\infty}\left(k_{n}-1\right)<\infty$. of $T$

We will prove that $\left\{x_{n}\right\}$ also $\triangle$-converges to a fixed point

Lemma 8. Let $X$ be a CAT(0) space, $C$ a closed convex subset of $X, T: C \rightarrow C$ an asymptotically nonexpansive mapping with coefficient $k_{n}$, and $\sum_{n=1}^{\infty}\left(k_{n}-1\right)<\infty$. If $F(T) \neq \emptyset$, $\left\{\alpha_{n}\right\},\left\{\beta_{n}\right\} \subseteq(0,1)$. Let $x_{0} \in C,\left\{x_{n}\right\}$ be generated by $x_{n+1}=$ $\alpha_{n} T^{n} x_{n} \oplus\left(1-\alpha_{n}\right) y_{n}, y_{n}=\beta_{n} x_{n} \oplus\left(1-\beta_{n}\right) T^{n} x_{n}, n \geq 0$. Then the limit $\lim _{n \rightarrow \infty} d\left(x_{n}, p\right)$ exists for all $p \in F(T)$.

Proof. Taking $p \in F(T)$, we have

$$
\begin{aligned}
d\left(x_{n+1}, p\right)= & d\left(\alpha_{n} T^{n} x_{n} \oplus\left(1-\alpha_{n}\right) y_{n}, p\right) \\
\leq & \alpha_{n} k_{n} d\left(x_{n}, p\right)+\left(1-\alpha_{n}\right) d\left(y_{n}, p\right) \\
\leq & \alpha_{n} k_{n} d\left(x_{n}, p\right) \\
& +\left(1-\alpha_{n}\right)\left\{\beta_{n} d\left(x_{n}, p\right)+\left(1-\beta_{n}\right) d\left(T^{n} x_{n}, p\right)\right\} \\
\leq & \alpha_{n} k_{n} d\left(x_{n}, p\right) \\
& +\left(1-\alpha_{n}\right)\left\{\beta_{n} d\left(x_{n}, p\right)+\left(1-\beta_{n}\right) k_{n} d\left(x_{n}, p\right)\right\} \\
= & \left\{1+\left(k_{n}-1\right)\left[1-\left(1-\alpha_{n}\right) \beta_{n}\right]\right\} d\left(x_{n}, p\right) .
\end{aligned}
$$

By Lemma 3, we can get that $\lim _{n \rightarrow \infty} d\left(x_{n}, p\right)$ exists.

Next, we will prove $\lim _{n \rightarrow \infty} d\left(T^{n} x_{n}, y_{n}\right)=0$.

Proposition 9. Let $X$ be a CAT(0) space, $C$ a closed convex subset of $X$, and $T: C \rightarrow C$ an asymptotically nonexpansive mapping with coefficient $k_{n}$. If $F(T) \neq \emptyset,\left\{\alpha_{n}\right\},\left\{\beta_{n}\right\} \subseteq(0,1)$. Let $x_{0} \in C,\left\{x_{n}\right\}$ be generated by $x_{n+1}=\alpha_{n} T^{n} x_{n} \oplus\left(1-\alpha_{n}\right) y_{n}, y_{n}=$ $\beta_{n} x_{n} \oplus\left(1-\beta_{n}\right) T^{n} x_{n}, n \geq 0$. Then under the hypotheses (H1) and (H2), one can get that $\lim _{n \rightarrow \infty} d\left(T^{n} x_{n}, y_{n}\right)=0$. 
Proof. By the assumption, $F(T)$ is nonempty. Take $p \in F(T)$, let $L=\sup _{n} k_{n}$, then we have

$$
\begin{gathered}
d\left(T^{n} x_{n}, p\right) \leq k_{n} d\left(x_{n}, p\right) \leq \operatorname{Ld}\left(x_{n}, p\right) \\
d\left(y_{n}, p\right) \leq \beta_{n} d\left(x_{n}, p\right)+\left(1-\beta_{n}\right) d\left(T^{n} x_{n}, p\right) \\
\leq \operatorname{Ld}\left(x_{n}, p\right) \\
d\left(T^{n} y_{n}, p\right) \leq k_{n} d\left(y_{n}, p\right) \leq L^{2} d\left(x_{n}, p\right) .
\end{gathered}
$$

It follows that the sequences $\left\{x_{n}\right\},\left\{T^{n} x_{n}\right\},\left\{y_{n}\right\},\left\{T^{n} y_{n}\right\}$ are bounded.

By Lemma 1, we have

$$
\begin{aligned}
d^{2}\left(x_{n+1}, p\right)= & d^{2}\left(\alpha_{n} T^{n} x_{n} \oplus\left(1-\alpha_{n}\right) y_{n}, p\right) \\
\leq & \alpha_{n} k_{n}^{2} d^{2}\left(x_{n}, p\right)+\left(1-\alpha_{n}\right) d^{2}\left(y_{n}, p\right) \\
& -\alpha_{n}\left(1-\alpha_{n}\right) d^{2}\left(T^{n} x_{n}, y_{n}\right) \\
\leq & d^{2}\left(x_{n}, p\right)+\left(1-\alpha_{n}\right)\left\{d^{2}\left(y_{n}, p\right)-d^{2}\left(x_{n}, p\right)\right\} \\
& +\alpha_{n}\left(k_{n}^{2}-1\right) d^{2}\left(x_{n}, p\right) \\
& -\alpha_{n}\left(1-\alpha_{n}\right) d^{2}\left(T^{n} x_{n}, y_{n}\right) .
\end{aligned}
$$

Similar to the proof of Proposition 6, we can get

$$
d^{2}\left(y_{n}, p\right)-d^{2}\left(x_{n}, p\right) \leq\left(1-\beta_{n}\right)\left(k_{n}^{2}-1\right) d^{2}\left(x_{n}, p\right) .
$$

Therefore, we have

$$
\begin{aligned}
d^{2}\left(x_{n+1}, p\right) \leq & d^{2}\left(x_{n}, p\right)+\left(1-\alpha_{n}\right)\left(1-\beta_{n}\right) \\
& \times\left(k_{n}^{2}-1\right) d^{2}\left(x_{n}, p\right) \\
& +\alpha_{n}\left(k_{n}^{2}-1\right) d^{2}\left(x_{n}, p\right) \\
& -\alpha_{n}\left(1-\alpha_{n}\right) d^{2}\left(T^{n} x_{n}, y_{n}\right) .
\end{aligned}
$$

Since $\left\{x_{n}\right\},\left\{y_{n}\right\}$ are bounded and $0<\delta<\alpha_{n}<1-\delta$ for all $n \geq n_{0}$. we have

$$
\begin{aligned}
\delta^{2} d^{2}\left(T^{n} x_{n}, y_{n}\right) \leq & d^{2}\left(x_{n}, p\right)-d^{2}\left(x_{n+1}, p\right) \\
& +\left(1-\alpha_{n}\right)\left(1-\beta_{n}\right)\left(k_{n}^{2}-1\right) d^{2}\left(x_{n}, p\right) \\
& +\alpha_{n}\left(k_{n}^{2}-1\right) d^{2}\left(x_{n}, p\right) .
\end{aligned}
$$

By the conditions (H1) and (H2), we have $\sum_{n=1}^{\infty}\left(k_{n}^{2}-1\right)<$ $\infty$ and

$$
\sum_{n=1}^{\infty} \delta^{2} d^{2}\left(T^{n} x_{n}, y_{n}\right)<\infty
$$

which implies that

$$
\lim _{n \rightarrow \infty} d^{2}\left(T^{n} x_{n}, y_{n}\right)=0
$$

Theorem 10. Let $X$ be a CAT(0) space, $C$ a closed convex subset of $X$, and $T: C \rightarrow C$ an asymptotically nonexpansive mapping with coefficient $k_{n}$. If $F(T) \neq \emptyset,\left\{\alpha_{n}\right\},\left\{\beta_{n}\right\} \subseteq(0,1)$. Let $x_{0} \in C,\left\{x_{n}\right\}$ be generated by $x_{n+1}=\alpha_{n} T^{n} x_{n} \oplus\left(1-\alpha_{n}\right) y_{n}, y_{n}=$ $\beta_{n} x_{n} \oplus\left(1-\beta_{n}\right) T^{n} x_{n}, n \geq 0$. Then under the hypotheses (H1) and (H2), one can get that $\left\{x_{n}\right\} \triangle$-converges to a fix point of $T$.

Proof. We first show that $\lim _{n \rightarrow \infty} d\left(x_{n}, T^{n} x_{n}\right)=0$. Indeed, by Lemma 1 , and $\beta_{n} \rightarrow 1$, we can get

$$
\begin{aligned}
d\left(x_{n}, y_{n}\right) & =d\left(x_{n}, \beta_{n} x_{n} \oplus\left(1-\beta_{n}\right) T^{n} x_{n}\right) \\
& \leq\left(1-\beta_{n}\right) d\left(x_{n}, T^{n} x_{n}\right) \longrightarrow 0 .
\end{aligned}
$$

And then,

$$
d\left(x_{n}, T^{n} x_{n}\right) \leq d\left(x_{n}, y_{n}\right)+d\left(y_{n}, T^{n} x_{n}\right) .
$$

By Proposition 9, we obtain that $\lim _{n \rightarrow \infty} d\left(x_{n}, T^{n} x_{n}\right)=0$.

We claim that $\lim _{n \rightarrow \infty} d\left(x_{n}, T x_{n}\right)=0$. Indeed we have

$$
\begin{aligned}
& d\left(x_{n+1}, x_{n}\right)= d\left(\alpha_{n} T^{n} x_{n} \oplus\left(1-\alpha_{n}\right) y_{n}, x_{n}\right) \\
& \leq \alpha_{n} d\left(T^{n} x_{n}, x_{n}\right)+\left(1-\alpha_{n}\right) d\left(x_{n}, y_{n}\right) \longrightarrow 0 \\
& d\left(x_{n}, T^{n-1} x_{n}\right) \leq d\left(\alpha_{n-1} T^{n-1} x_{n-1} \oplus\left(1-\alpha_{n-1}\right) y_{n-1}, T^{n-1} x_{n}\right) \\
& \leq \alpha_{n-1} d\left(T^{n-1} x_{n-1}, T^{n-1} x_{n}\right) \\
&+\left(1-\alpha_{n-1}\right) d\left(y_{n-1}, T^{n-1} x_{n}\right) \\
& \leq \alpha_{n-1} k_{n-1} d\left(x_{n-1}, x_{n}\right) \\
&+\left(1-\alpha_{n-1}\right)\left[d\left(y_{n-1}, T^{n-1} x_{n-1}\right)\right. \\
&\left.+d\left(T^{n-1} x_{n-1}, T^{n-1} x_{n}\right)\right] \\
& \leq \alpha_{n-1} k_{n-1} d\left(x_{n-1}, x_{n}\right) \\
&+\left(1-\alpha_{n-1}\right)\left[d\left(y_{n-1}, T^{n-1} x_{n-1}\right)\right. \\
&\left.+k_{n-1} d\left(x_{n-1}, x_{n}\right)\right] \longrightarrow 0
\end{aligned}
$$

Thus,

$$
\begin{aligned}
d\left(x_{n}, T x_{n}\right) & \leq d\left(x_{n}, T^{n} x_{n}\right)+d\left(T^{n} x_{n}, T x_{n}\right) \\
& \leq d\left(x_{n}, T^{n} x_{n}\right)+\operatorname{Ld}\left(T^{n-1} x_{n}, x_{n}\right) \longrightarrow 0 .
\end{aligned}
$$

Since $\left\{x_{n}\right\}$ is bounded, we may assume that $\left\{x_{n}\right\} \triangle$ converges to a point $\widehat{x}$. By Lemma 2 , we have $\widehat{x} \in F(T)$.

\section{Acknowledgment}

This research was supported by NSFC Grants nos. 11071279 and 11226125 .

\section{References}

[1] K. Goebel and W. A. Kirk, "Topics in metric fixed point theory," in Cambridge Studies In Advanced Mathematics, vol. 28, Cambridge University Press, Cambridge, UK, 1990. 
[2] K. Goebel and W. A. Kirk, "A fixed point theorem for asymptotically nonexpansive mappings," Proceedings of the American Mathematical Society, vol. 35, pp. 171-174, 1972.

[3] J. Schu, "Approximation of fixed points of asymptotically nonexpansive mappings," Proceedings of the American Mathematical Society, vol. 112, no. 1, pp. 143-151, 1991.

[4] J. Górnicki, "Weak convergence theorems for asymptotically nonexpansive mappings in uniformly convex Banach spaces," Commentationes Mathematicae Universitatis Carolinae, vol. 30, no. 2, pp. 249-252, 1989.

[5] W. A. Kirk, "Geodesic geometry and fixed point theory. II," in International Conference on Fixed Point Theory and Applications, pp. 113-142, Yokohama Publishers, Yokohama, Japan, 2004.

[6] B. Halpern, "Fixed points of nonexpanding maps," Bulletin of the American Mathematical Society, vol. 73, pp. 957-961, 1967.

[7] K.-K. Tan and H. K. Xu, "Fixed point iteration processes for asymptotically nonexpansive mappings," Proceedings of the American Mathematical Society, vol. 122, no. 3, pp. 733-739, 1994.

[8] L. Y. Shi and R. D. Chen, "Strong convergence of viscosity approximation methods for nonexpansive mappings in CAT(0) spaces," Journal of Applied Mathematics, vol. 2012, Article ID 421050, 11 pages, 2012.

[9] S. Dhompongsa and B. Panyanak, "On $\delta$-convergence theorems in CAT(0) spaces," Computers \& Mathematics with Applications, vol. 56, no. 10, pp. 2572-2579, 2008.

[10] P. Chaoha and A. Phon-on, "A note on fixed point sets in CAT(0) spaces," Journal of Mathematical Analysis and Applications, vol. 320, no. 2, pp. 983-987, 2006.

[11] S. Dhompongsa, W. A. Kirk, and B. Sims, "Fixed points of uniformly Lipschitzian mappings," Nonlinear Analysis: Theory, Methods \& Applications, vol. 65, no. 4, pp. 762-772, 2006.

[12] W. A. Kirk and B. Panyanak, "A concept of convergence in geodesic spaces," Nonlinear Analysis: Theory, Methods \& Applications, vol. 68, no. 12, pp. 3689-3696, 2008.

[13] N. Hussain and M. A. Khamsi, "On asymptotic pointwise contractions in metric spaces," Nonlinear Analysis: Theory, Methods \& Applications, vol. 71, no. 10, pp. 4423-4429, 2009.

[14] S.-s. Chang, Y. J. Cho, and H. Zhou, "Demi-closed principle and weak convergence problems for asymptotically nonexpansive mappings," Journal of the Korean Mathematical Society, vol. 38, no. 6, pp. 1245-1260, 2001.

[15] K.-K. Tan and H. K. Xu, "The nonlinear ergodic theorem for asymptotically nonexpansive mappings in Banach spaces," Proceedings of the American Mathematical Society, vol. 114, no. 2, pp. 399-404, 1992. 


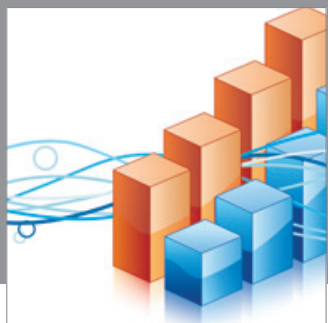

Advances in

Operations Research

mansans

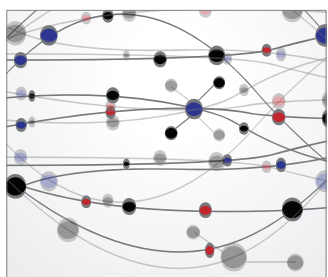

The Scientific World Journal
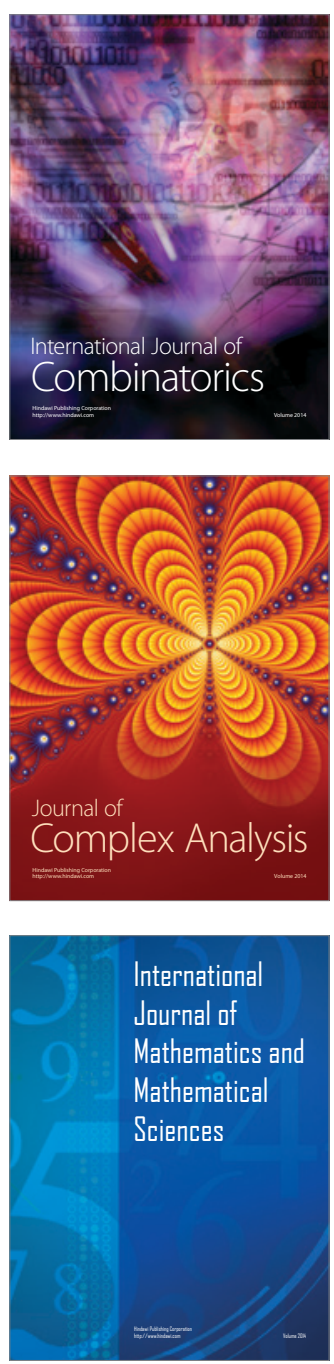
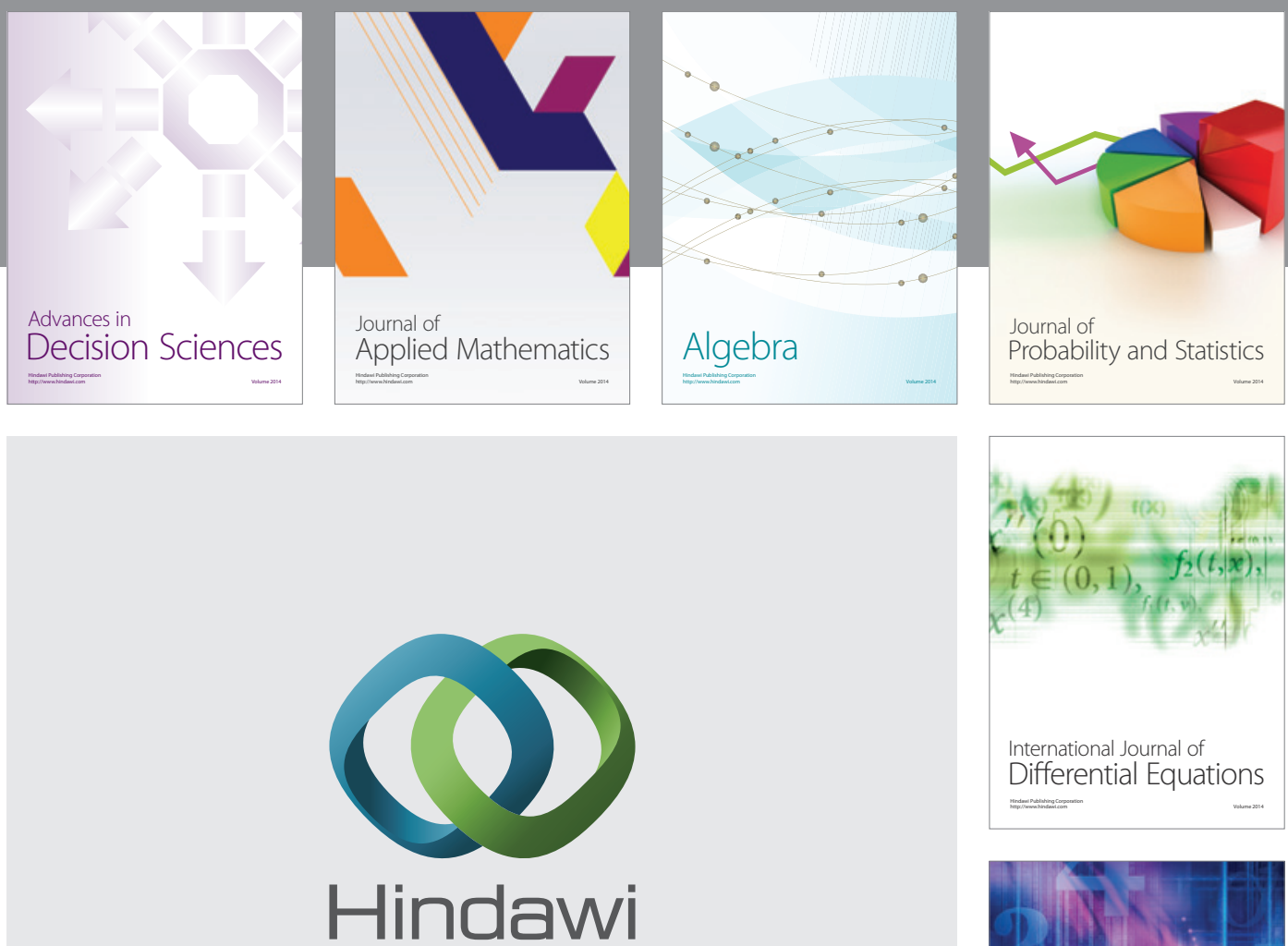

Submit your manuscripts at http://www.hindawi.com
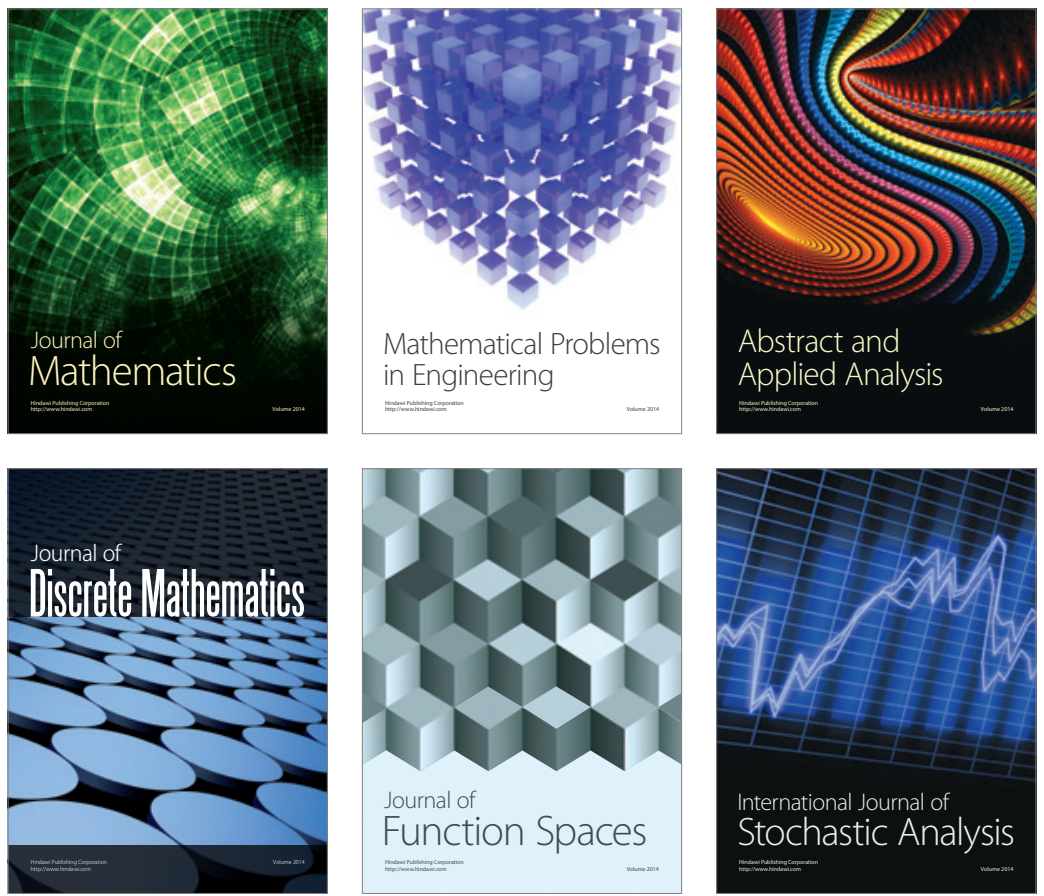

Journal of

Function Spaces

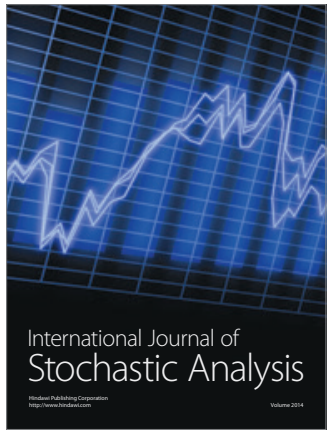

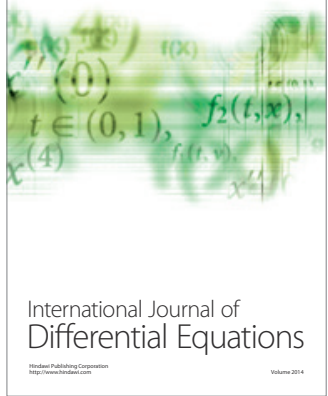
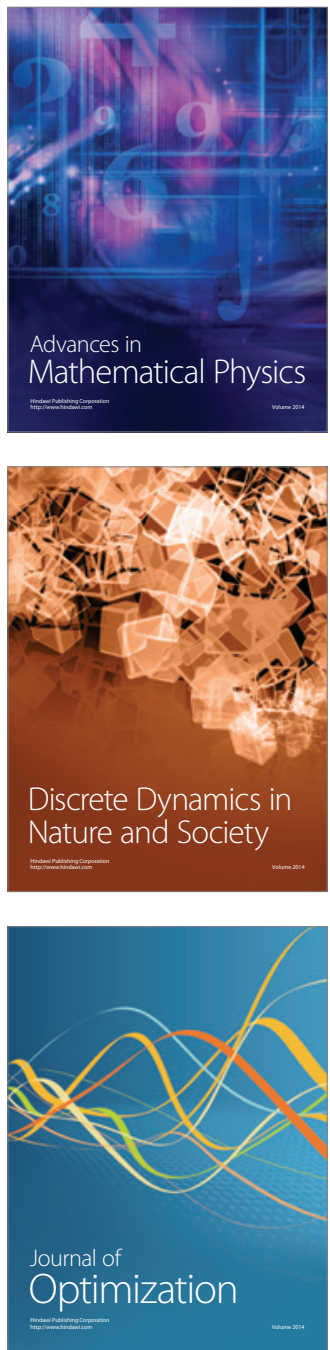